\title{
In praise of maize
}

\section{The field of genetics owes its existence and most of its methods to agriculture. This year, genomic strategies and tools have notably begun to pay back the favor. Crop plants may be not only the discipline's most readily translated applications but also its most fruitful model organisms.}

A ert readers may have realized by now that for the last quarter of this year, all three covers of the journal have been devoted to agricultural plant genetics. We have the content to fill those wrappers, along with readership download data that persuade us that these articles are every bit as interesting to our readers as those about biomedical and model animal studies that accompany them.

The field of genetics received its name from William Bateson at the Third International Conference on Plant Hybridization in London, England in 1906 (Wilks, W. Report of the Third 1906 International Conference on Genetics: hybridisation (the cross-breeding of genera or species), the cross-breeding of varieties, and general plant breeding, Royal Horticultural Society, London, UK, 1907). The discipline got its next big intellectual boost from crops following World War I in 1919 when R.A. Fisher developed the analysis of variance strategy at the Rothamsted plant breeding facility and produced the statistical toolkit underpinning most current genetic methods (Fisher, R.A. Statistical Methods for Research Workers, Oliver and Boyd, Edinburgh, Scotland, 1925).

The current set of agricultural genetics studies results from the efforts of internationally excellent institutions and collaborations, frequently reflecting China's long history of expertise in plant breeding and its recent investment in genomic tools. Jianming Yu et al. developed a method to deal simultaneously with both linkage and association data and applied this method to both maize and human pedigrees (Nat. Genet. 38, 203-208, 2005). In our November issue, Jinsheng Lai et al. (Nat. Genet. 42, 1027-1030, 2010) made tractable the massive and highly polymorphic maize genome by characterizing genetic variation in the six elite strains most commonly used to make commercial hybrid crops. In the same issue, Bin Han and colleagues delivered a linkage disequilibrium map of rice from 517 landraces, which allowed them to carry out SNP tagging and association analysis of 14 traits (Nat. Genet. 42, 961-967, 2010). Now, Hon-Ming Lam et. al. (pages 1053-1059) have sequenced the diversity of 31 soybean genomes to demonstrate the extensive genome-wide linkage disequilibrium pattern that can be used both to select hybrids and to cross in new traits from the much greater diversity of mutually fertile wild beans.

Genome mapping for breeding better crops will be aided by a systems-level conception of the networks of photosynthetic production, leaf development and biomass synthesis. To this end, Thomas Brutnell and colleagues (pages 1060-1067) have characterized global gene expression in a number of constituent cell types in the maize leaf. Topics in this same issue of the journal range from cell- and organism-level mechanisms of development to the structures and mechanisms of populations. Plant populations offer both the numbers and the controlled geographic and environmental distributions for us to begin to study the genetic architecture of populations. Within these systems, mechanisms of evolution can be seen that relate gene-gene interactions to differential fitness. For example, on pages $1135-1139$ of this issue, Matthieu Reymond and colleagues report that geographic patterns of reproductive incompatibility in wild Arabidopsis populations can be driven by allelic incompatibility that results from differential disease sensitivity in genes encoding immune signaling molecules.

Genetics is an integrative area of study, and it is worth taking the time to consider the whole range of skills it teaches, from sequencing recombinant DNA to cytogenetics to statistical models of population evolution. We believe that publishing a robust mixture of current research is stimulating. The discipline is at its most productive when strategies grown in one crop cross-fertilize other fields. 